\title{
Actitudes y concepciones socioeducativas hacia jóvenes con diversidad funcional intelectual en el contexto escolar andaluz
}

\author{
Socio-Educational Attitudes and Conceptions About the Youngs With Intellectual Functional \\ Disability in the Andalusian School Context
}

\section{Atitudes e concepções socioeducativas sobre jovens com diversidade funcional intelectual no ambiente escolar andaluz}

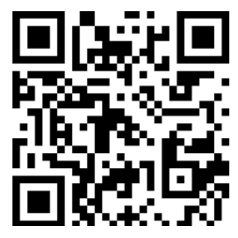

\author{
María Isabel Negri-Cortés \\ Universidad Internacional de la Rioja \\ Málaga, España \\ mariaisabel.negri@unir.net \\ https://orcid.org/0000-0003-0548-2187 \\ Juan José Leiva-Olivencia \\ Universidad de Málaga \\ Málaga, España \\ juanleiva@uma.es \\ https://orcid.org/0000-0002-2857-8141
}

Recibido • Received • Recebido: 06 / 11 / 2019

Corregido • Revised • Revisado: 07 / 07 / 2021

Aceptado • Accepted • Aprovado: 04 / 08 / 2021

\begin{abstract}
Resumen:
Introducción. Las relaciones personales de la juventud con diversidad funcional intelectual (en adelante DFI) en los diferentes ámbitos de su vida escolar y personal son determinantes para su plena inclusión. Objetivo. Analizar las concepciones y actitudes socioeducativas que existen actualmente hacia el alumnado con DFI. Metodología. Se utilizó un diseño mixto de investigación, para el cual contamos con una muestra incidental de 38 docentes de seis centros de educación secundaria y 37 profesionales de nueve entidades sociales de apoyo a la inclusión de jóvenes con DFI de Málaga. La recogida de datos se realizó mediante dos cuestionarios ad hoc y un total de 18 entrevistas a diferentes agentes educativos del ámbito formal y no formal. Resultados. Existen ciertas dificultades en el sistema educativo para atender la diversidad; se delega la atención de este alumnado a profesionales de apoyo a la integración; las relaciones interpersonales de esta juventud suelen darse con otros compañeros y compañeras con necesidades educativas especiales (en adelante NEE), y son muy reducidas o basadas en burlas y acoso. El trato paternalista del profesorado dificulta su desarrollo autónomo e incide negativamente en su autoestima. Discusión. Se propone promover la autodeterminación de esta juventud, concienciando y sensibilizando a la comunidad educativa hacia concepciones positivas de la diversidad.
\end{abstract}

Palabras claves: Deficiencia mental; educación compensatoria; igualdad de oportunidades; integración social. 
http://doi.org/10.15359/ree.25-3.23

http://www.una.ac.cr/educare

educare@una.ac.cr

\begin{abstract}
:
Introduction. Personal relationships of young people with intellectual functional diversity (IFD) in their different school contexts and personal life are essential for their full inclusion. Objective. Analyze the social and educational conceptions and attitudes existing, nowadays, in relation to students with IFD. Methodology. We used a mixed research design, for which we had an incidental sample of 38 teachers from six secondary education schools and 37 professionals from nine social entities of Málaga. The data were collected by two ad-hoc questionnaires and 18 interviews to formal and nonformal educational agents. Results. The main results show the difficulties of the educational system to attend to diversity. The attention of these students is delegated to professionals supporting the integration. The interpersonal relationships of these young people are usually with other peers with special educational needs (SEN) and are either very limited or based on mockery and bullying. Paternal behavior of the teachers hinders their autonomy and, as a result, we saw that these young have low self-esteem. Discussion. we propose to improve the self-determination of these young people by raising awareness within the educational community for better conceptions about diversity.
\end{abstract}

Keywords: Intellectual disability; compensatory education; equal opportunity; social integration.

\begin{abstract}
Resumo:
Introdução. As relações pessoais dos jovens com Diversidade Funcional Intelectual (DFI) nas diferentes áreas da sua vida educativa e pessoal são decisivas para a sua plena inclusão. Objetivo. Analisar as concepções socioeducativas e atitudes que atualmente existem em relação aos estudantes com DFI. Metodologia. Foi utilizado um desenho de investigação mista, para o qual temos uma amostra incidental de 38 professores de seis colégios e 37 profissionais de nove entidades sociais em Málaga. A coleta de dados foi realizada por meio de dois questionários adhoc e um total de 18 entrevistas com diferentes agentes educacionais no campo formal e não formal. Resultados. Os principais resultados mostram as dificuldades do sistema educacional no atendimento à diversidade, que delega a atenção desses estudantes aos profissionais que apoiam a integração, e que as relações interpessoais desses jovens tendem a ser com outros companheiros com Necessidades Educativas Especiais (NEE); são muito reduzidas e baseadas em provocações e assédio. $O$ tratamento paternalista do corpo docente dificulta seu desenvolvimento autônomo, afetando negativamente sua autoestima. Discussão. Propõe-se promover a autodeterminação desses jovens, sensibilizandoos e sensibilizando a comunidade educacional para concepções positivas de diversidade.
\end{abstract}

Palavras-chave: Deficiência mental; educação compensatória; igualdade de oportunidades; integração social.

\title{
Introducción
}

La llegada del alumnado con DFI a los centros educativos ordinarios supone un gran avance para su inclusión al compartir currículo, recursos y espacios de interacción con sus iguales. No obstante, tras décadas de exclusión, la presencia de este alumnado puede llevar consigo dificultades ancladas en la estigmatización de sus características personales, que podrían dificultar su atención educativa y relaciones sociales, y afectar su desarrollo socioemocional y educativo. 
En este sentido, resulta especialmente susceptible el alumnado que se encuentra cursando la etapa de Educación Secundaria Obligatoria (ESO), y estudios postobligatorios, como los ciclos de Formación Profesional Básica (FPB) (Jefatura del Estado, 2013). Esto se debe a las edades del alumnado (12-18 años) ya que, la adolescencia es una etapa de desarrollo de la independencia social y de autoafirmación, donde la aceptación del grupo de iguales es fundamental para el desarrollo de la autoestima y el crecimiento social. En este momento vital, dadas sus características y dificultades personales, el alumnado con DFI podría padecer especialmente las consecuencias de prejuicios y concepciones sociales erróneas, que podrían perjudicar su desarrollo autónomo y social (Verdugo Alonso y Rodríguez Aguilella, 2012). La exclusión y estigmatización social del colectivo se manifiesta también a través de las estrategias organizativas y metodológicas de la institución educativa y en la concepción socio-educativa que presentan los agentes educativos hacia estos grupos de jóvenes (López et al. 2010).

El objetivo del presente estudio ha sido conocer las actitudes y concepciones existentes en la actualidad sobre la realidad socio-educativa del alumnado con DFI.

\section{Actitudes y concepciones socioeducativas frente a la plena inclusión del alumnado con DFI}

La Ley Orgánica 2/2006, de 3 de mayo (Jefatura del Estado, 2006) junto con la actual Ley Orgánica 8/2013, de 9 de diciembre (Jefatura del Estado, 2013) sientan el marco legislativo de la atención educativa al alumnado con DFI, ubicándoles en el grupo de estudiantes con necesidades educativas especiales (NEE). Al mismo tiempo, la Orden de 25 de julio de 2008 (Consejería de Educación, 2008) establece las medidas de atención a la diversidad del alumnado con necesidades específicas de apoyo educativo (NEAE) a desarrollar en los centros educativos andaluces. Así, la inclusión y la atención multidisciplinar serán los principios que deberán regir la atención educativa al alumnado con NEAE, donde la acción tutorial juega un papel primordial en esta labor. Las principales actuaciones para la atención a la diversidad del alumnado se centran en proponer estrategias organizativas y metodológicas, y prestar apoyos y adaptaciones curriculares.

Una de las más relevantes medidas de atención a la diversidad que se proponen desde la Ley Orgánica 2/2006 (Jefatura del Estado, 2006) tiene que ver con la creación de itinerarios educativos de carácter compensatorio como los Programas de Cualificación Profesional Inicial (PCPI) que tienen como objetivo facilitar el tránsito del estudiantado a la vida activa y laboral, mediante el desarrollo de competencias profesionales (García-Ruiz y Sánchez Muñoz, 2012). Actualmente, estos programas tienen su equivalente en los ciclos de FPB implantados desde la Ley Orgánica 8/2013 (Jefatura del Estado, 2013).

Todas las medidas anteriormente mencionadas pretenden impulsar el desarrollo integral del alumnado con DFl, pero de acuerdo con Verdugo Alonso et al. (2018), las principales limitaciones de los mecanismos de atención a la diversidad actuales surgen de la excesiva 
http://doi.org/10.15359/ree.25-3.23

http://www.una.ac.cr/educare

educare@una.ac.cr

atención que se presta desde la misma a los aspectos curriculares, la descontextualización de los procesos de evaluación de necesidades y la carencia de una mayor competencia por parte de profesionales responsables de esta.

Se hace necesario, por tanto, actuar desde la propia cultura del centro educativo, centrando la atención en las actitudes y concepciones socioeducativas que existen sobre el alumnado con DFI, dado que esto podría favorecer su proceso de inclusión (Luque-Parra y Luque-Rojas, 2011). A tal efecto, coincidimos con las aportaciones de diversos estudios como los de Moneo Estany y Anaut Bravo (2017), Macías Gómez (2016) y Verdugo (como se citó en Verdugo Alonso y Rodríguez Aguilella, 2012) en señalar el carácter educable de las actitudes a partir de la modificación de las conductas, partiendo de la formación y la reconstrucción de significados. De esta manera, podrían superarse estereotipos negativos y promover nuevas concepciones socioeducativas hacia las personas con DFI, que favorezcan la igualdad y promocionen el respeto a la diversidad.

En la modificación de actitudes en el centro educativo y la transformación de las concepciones socioeducativas acerca del alumnado con DFI, el profesorado juega un papel fundamental. Así, una actitud positiva del personal docente hacia este alumnado contribuye a flexibilizar y diversificar los contenidos curriculares con base en sus necesidades e intereses (Barría Rojas y Jurado de los Santos, 2016).

No obstante, siguiendo las investigaciones de autoras como Vilà Suñé et al. (2012) y Polo Sánchez y Aparicio Puerta (2018), una de las principales dificultades que existen para que esto ocurra surge de la falta de formación del personal docente, cuya escasa sensibilización hacia la diversidad del alumnado puede incidir directamente en la conducta manifestada por el grupo de iguales. Esta falta de capacitación también puede derivar en una carente atención hacia el alumnado con DFI en el aula ordinaria (Verdugo Alonso y Rodríguez Aguilella, 2012), o al hecho de que el personal docente prefiera la presencia en el aula del maestro o maestra en educación especial, o en la segregación de estos grupos al aula de apoyo a la integración (Pegalajar Palomino y Colmenero Ruiz, 2017). En definitiva, la falta de formación del profesorado puede generar que este evada su responsabilidad en el desarrollo del alumnado con DFI, y generar una mayor distancia curricular y socioeducativa respecto al resto de estudiantes.

Partiendo de las situaciones anteriormente expresadas, debemos revisar investigaciones como las de Suriá Martínez (2011), que indagan en las relaciones interpersonales del alumnado con DFI y sus iguales en los niveles de ESO y en estudios superiores, determinando la presencia de actitudes poco favorables en el estudiantado de secundaria, donde los últimos cursos de esta etapa educativa parecen ser los más conflictivos. Esta visión coincide con la de Moneo Estany y Anaut Bravo (2016), que también detectan actitudes poco inclusivas en el alumnado universitario relacionadas con la pena, la sobreprotección o la exclusión, debidas a la falta de sensibilización y visibilización del alumnado con DFI en las etapas obligatorias. 
Las dificultades sociales del alumnado con DFI son especialmente relevantes al final de la educación obligatoria, pues las consecuencias de su aislamiento social podrían derivar en una baja autoestima, acoso escolar e incluso fracaso o abandono escolar temprano, que limita, así, sus oportunidades de inclusión en nuevos contextos sociales, como es el mercado laboral. Prestando atención a esta cuestión, nos apoyamos en Moneo Estany y Anaut Bravo (2016), que identifican la convivencia como eje determinante de la sensibilización y normalización de este alumnado en cualquier contexto social. Igualmente, Luque-Parra y Luque-Rojas (2011), señalan, además, el carácter transformador de la convivencia en las actitudes del alumnado hacia sus iguales con DFI.

A tal efecto, apostar por la participación del alumnado con DFI en el aula ordinaria, compartiendo espacios de aprendizaje y relaciones interpersonales con sus compañeros, contribuirá a favorecer su normalización y mejorar las concepciones socioeducativas que existen respecto a ellos. Así, el proceso de aprendizaje de este alumnado no solo debe centrarse en su desarrollo académico e intelectual, sino también en su educación emocional y relaciones interpersonales (Rodríguez González, 2017). Con este fin, es necesario actuar no solo desde la intervención al alumnado con DFI, sino también desde las actitudes y concepciones socioeducativas que manifiestan los diferentes agentes del centro educativo, donde el profesorado y el grupo de iguales juegan un papel fundamental.

Por tanto, debemos entender que atender a la diversidad del alumnado debe ser responsabilidad de toda la comunidad educativa (ArnaizSánchezet al., 2018) y así debe plasmarse en la organización, funcionamiento y principios de los centros educativos, promoviendo una transformación constructiva, ética y cultural.

\section{Método}

\section{Objetivos}

El objetivo general de este estudio de investigación es conocer las actitudes y concepciones socioeducativas que se manifiestan actualmente hacia la juventud con DFI desde los contextos educativos y formativo-laborales que frecuentan. Los objetivos específicos son: a) Conocer los elementos organizativos y estructurales del sistema educativo que perpetúan la segregación de los jóvenes y las jóvenes con DFl; b) Indagar en las relaciones interpersonales que desarrollan los jóvenes y las jóvenes con DFl; c) Descubrir la repercusión de los estereotipos sociales sobre este colectivo en los agentes educativos, especialmente el profesorado; y d) Analizar la influencia de las situaciones de segregación que viven los jóvenes y las jóvenes en su desarrollo autónomo.

\section{Procedimiento y diseño de investigación}

Se ha utilizado un modelo mixto de investigación que nos otorga la oportunidad de acercarnos más profundamente al problema de investigación, ofreciéndonos una mayor comprensión de la realidad de los fenómenos sociales. En la parte cualitativa de nuestra 
http://doi.org/10.15359/ree.25-3.23

http://www.una.ac.cr/educare

educare@una.ac.cr

investigación, desarrollamos dos estudios de casos, que nos permiten analizar la información obtenida desde un enfoque interpretativo y heurístico (Ceballos-Herrera, 2009), construyendo la realidad de los contextos seleccionados, facilitando el conocimiento en profundidad de los procesos, situaciones y agentes implicados en los contextos seleccionados.

\section{Contexto y muestra}

El objeto de nuestro estudio en su vertiente cualitativa se centra en la realidad acontecida en dos contextos socioeducativos de los ámbitos educativo y formativo-laboral de la provincia de Málaga (España), se analizan las percepciones y opiniones de los agentes implicados sobre su labor profesional.

En primer lugar, hemos seleccionado desde el ámbito de la educación formal los ciclos de FPB (PCPI en el momento de nuestra investigación) de NEE de un centro de Educación Secundaria (IES Concepción Villalba), que garantiza una atención a la diversidad de óptima calidad para su alumnado en las etapas educativas obligatorias y postobligatorias que oferta, gracias a una amplia dotación en recursos materiales y humanos especializados. Este centro oferta tres itinerarios de FPB desde los cuales se atienden grupos 8 a 10 estudiantes de 18 a 22 años, que presentan NEE y que cuentan con un nivel considerable de autonomía personal. Participaron 5 profesionales de la educación que atienden a estos grupos de jóvenes: tres docentes de taller, una de las orientadoras del centro y una maestra en pedagogía terapéutica, dos alumnas de los ciclos de FPB y sus familias.

Desde el ámbito de las entidades sociales, seleccionamos una asociación promotora de la inclusión de las personas con DFI en nuestra sociedad (Asociación La Unión), que cuenta con diversos programas y servicios, como un Centro Ocupacional, un Centro Especial de Empleo y un Servicio de Formación y Empleo. En este caso participaron tres profesionales del ámbito social: la responsable del servicio de formación y empleo, la técnica de orientación y promoción laboral del centro ocupacional y una de las monitoras de taller, así como 4 personas usuarias de sus diferentes programas y dos familiares del público usuario.

Respecto a la recogida de información cuantitativa, participaron profesionales del ámbito educativo formal y no formal de la provincia de Málaga.

En el ámbito formativo-escolar, contamos con profesionales de seis centros educativos de Educación Secundaria que cuentan con recursos y programas para la atención a la diversidad del alumnado con NEE, ofertan además cuatro de ellos ciclos de FPB específicos. Contamos con 38 participantes, de los cuales un 36,8\% son docentes de formación profesional, un 28,9\% son profesionales de apoyo a la integración, un 18,4\% son docentes de ESO, un 13,2\% son profesionales de la orientación educativa y un $2,6 \%$ son profesionales de otras especialidades educativas. 
Desde el ámbito socio-laboral, los grupos de profesionales pertenecían a nueve entidades sociales que atienden la formación e inserción sociolaboral de jóvenes con DFI. En este ámbito, el número de participantes es de 37 , donde un $45,9 \%$ son profesionales de apoyo al ajuste personal y social, un $24,3 \%$ son docentes de taller ocupacional, un $13,5 \%$ son de preparación laboral, un $10,8 \%$ son de tecnología de la formación para el empleo y un $5,4 \%$ son de orientación laboral.

Para alcanzar los consiguientes objetivos se ha llevado a cabo un muestreo de tipo incidental, en tanto que el personal investigador es el que se ha encargado de llevar a cabo la elección de los sujetos que debían formar parte de la muestra, tratando de reunir los casos estimados más representativos de la población. Los criterios de elección se han fundamentado en el conocimiento teórico de que disponen sobre la temática central de este estudio y los supuestos previos en relación con la población, cuya validez muestral presenta un 95\% de confianza y un margen de error inferior al $10 \%$.

\section{Instrumentos de recogida de información}

En la parte cualitativa de la investigación, hemos optado por la realización de entrevistas semiestructuradas, que nos han permitido conocer las experiencias y percepciones de los sujetos participantes, acercándonos a la realidad acontecida en cada uno de los contextos. Dada la diversidad de las personas participantes se diseñaron dos guiones de entrevistas: uno dirigido a las personas profesionales y otro a familiares, en los que se formularon cuestiones relativas a la trayectoria educativa del alumnado/público usuario, su formación para el empleo y también sobre la inclusión social y laboral de las personas con DFI.

Para la parte cuantitativa de nuestro estudio diseñamos dos cuestionarios, para cada uno de los contextos a los que nos dirigimos (educativo y socio-laboral). Cada cuestionario consta de 30 ítems formulados en escala Likert, en los que indagamos en las actitudes y percepciones que tienen los grupos profesionales de cada uno de los ámbitos referidos. Cada cuestionario está dividido en dos partes: una primera parte compuesta de seis cuestiones sobre las características personales de los sujetos participantes, y una segunda parte, formada por 24 ítems, donde se profundiza en los aspectos más significativos de cada uno de los ámbitos de estudio.

Previo a la aplicación de los instrumentos, se realizó un estudio de sus características psicométricas y se recogieron evidencias sobre la fiabilidad y la validez de la medida que proporcionaba. Fueron validados por 5 personas expertas en metodología de investigación, pertenecientes a varios departamentos de Educación de la Universidad de Málaga sin relación con la investigación. Asimismo, se comprobó que presentaban una consistencia interna aceptable (a de Cronbach de .887, educativo, y .889, el socio-laboral). Para la validez de constructo se realizaron análisis factoriales categoriales de componentes principales (CATPCA), teniendo en cuenta la naturaleza combinada de la escala Likert utilizada en ambos cuestionarios. 
http://doi.org/10.15359/ree.25-3.23

http://www.una.ac.cr/educare

educare@una.ac.cr

\section{Análisis}

En el análisis de los datos obtenidos en nuestro estudio, hemos recurrido a una estrategia de triangulación de datos que nos otorgan una visión ampliada de la realidad objeto de nuestra investigación.

Para el tratamiento de la información extraída mediante nuestros cuestionarios, hemos utilizado el programa de análisis estadístico S.P.S.S. 12.0. Para la categorización de la información obtenida en nuestros estudios de caso, hemos utilizado el programa Nudist-Vivo 8.0, llegando a extraer hasta 10 categorías generales en cada caso. A partir de la codificación y clasificación de los datos, hemos podido realizar la triangulación de estos mismos, y se llegó a establecer relaciones, comparaciones y reflexiones pertinentes sobre estos.

\section{Resultados}

\section{Sobre la organización del sistema educativo}

En muchas ocasiones, la propia configuración del sistema educativo es la principal responsable de perpetuar situaciones de discriminación y segregación del alumnado con DFI. Si analizamos cómo es la atención que recibe este alumnado en los centros educativos ordinarios, nos encontramos situaciones como la que describe esta docente.

Las clases son muy numerosas hoy en día. Si tienes dos alumnos, o uno incluso, con NEE, entonces tienes dos opciones: o abandonas un poco al resto de la clase y te dedicas a ese alumno, o lo contrario, ese chico está ahí, pero tú no le puedes dar todas las atenciones que quisieras darle como docente. (Entrevista a ... profesora de taller PCPI Marroquinería). (Negri Cortés, 2016, p. 317)

Prestando atención a cuestiones organizativas y estructurales, podemos observar las dificultades a las que deben enfrentarse el personal profesional educativo para atender a la diversidad del alumnado dentro del aula. Por un lado, existe una elevada ratio de estudiantes por grupo clase, que dificulta la atención individualizada a sus necesidades. El personal docente no se siente capacitado para proporcionar una atención personalizada y especializada al alumnado con DFI, por lo que termina optando por el abandono educativo de este alumnado o delegando su atención educativa a los grupos profesionales especializados.

Por otro lado, la diferenciación realizada entre el alumnado con NEE y el resto, nos habla de una ideología homogeneizadora existente en los centros educativos que gira en torno al concepto de normalidad. Esta catalogación puede acrecentar las distancias de este alumnado hacia su proceso de inclusión en los contextos socio-educativos. Como consecuencia de estas dos situaciones se constituye una percepción del alumnado con DFI desde el ámbito educativo basada en sus dificultades y no en sus potencialidades, este alumnado con DFI es tratado como 
http://doi.org/10.15359/ree.25-3.23

un obstáculo para la labor docente y para el aprendizaje de sus compañeros y compañeras, y no como una oportunidad de enriquecimiento social.

Para atender las necesidades individuales socio-educativas del alumnado con DFI, desde el sistema educativo se ofertan diferentes medidas de carácter compensatorio. A este efecto, hemos indagado en la percepción del personal docente respecto a si el alumnado con DFI debe recibir apoyo individualizado del personal del departamento de orientación varias horas a la semana. Como resultado, como se muestra en la Figura 1, hemos podido conocer que un 45,9\% piensa que sí, porque hay algunas actividades para las que necesitan un apoyo específico, mientras un $43,2 \%$ considera que sí, siempre que las actividades que realicen guarden coherencia con las competencias que se trabajan en clase. Por otro lado, un 5,4\% manifiesta que no es necesario, pero el personal de este departamento debe preparar actividades para que el alumnado trabaje en clase y un 5,4\% piensa que no, porque esta es una práctica segregadora (Negri Cortés, 2016).

Figura 1: La juventud con Diversidad Funcional Intelectual deben recibir apoyo del personal del departamento de orientación varias horas a la semana

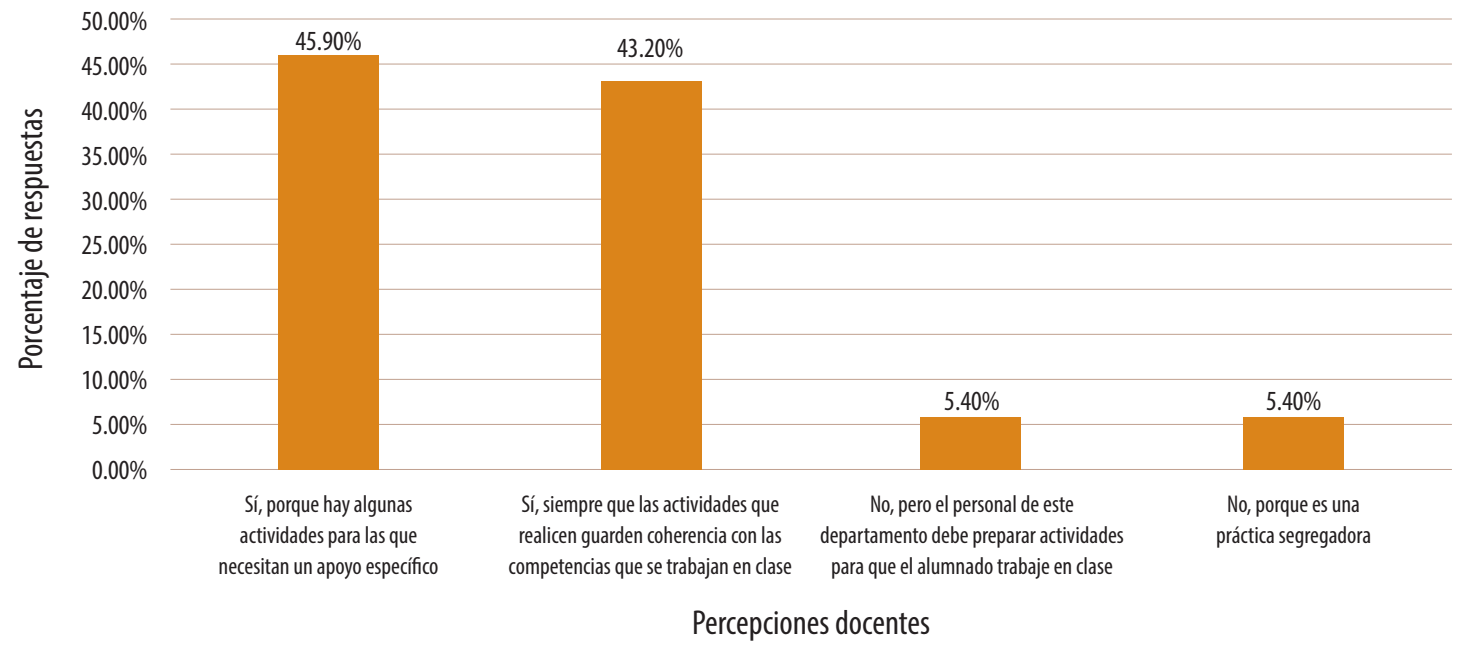

Nota: Elaboración propia.

Con base en los resultados obtenidos, existe una visión positiva en el profesorado respecto a las funciones de apoyo que realizan estos grupos profesionales. La presencia en el centro educativo de especialistas en la atención a la diversidad resulta ser un potencial valor para satisfacer las necesidades educativas del alumnado que así lo requiere. No obstante, desde las escuelas se puede caer en malas prácticas que deleguen por completo la atención educativa de este alumnado en este tipo de profesional, que suele ser fuera del aula ordinaria. Por tanto, el desarrollo socioeducativo del alumnado con DFI se produce de manera separada de sus pares 
http://doi.org/10.15359/ree.25-3.23

http://www.una.ac.cr/educare

educare@una.ac.cr

de aulas, sin interacciones directas entre todo el alumnado, presenten o no NEE, además de no compartir un mismo contexto de aprendizaje. Esto incrementa aún más la distancia social que separa a este colectivo de los procesos educativos ordinarios y de unas relaciones sociales plenamente inclusivas.

\section{Sobre las relaciones interpersonales}

La constitución de relaciones interpersonales de afecto y amistad resulta esencial para favorecer la normalización y visibilización de las personas con DFI en nuestra sociedad. No obstante, este alumnado en ocasiones pueden tener dificultades para relacionarse con sus iguales, especialmente en su adolescencia.

Cuando ya entró en el instituto, date cuenta que era una edad de 15 o 16 años, y entró en el instituto y aquello fue horrible. Él es un niño hiperactivo, es un niño que tiene sus problemas, todo, entonces lo pasamos mal. Con esa edad, pues es normal, le gustan las niñas, bueno, con esa edad y ahora es normal. Entonces claro, los demás, a él le gusta, eh, que yo no lo estoy excusando, le decían "pues levántale la falda" y él lo hacía y en casa le decíamos: "pero bueno J. S., ¿cómo puedes hacer esas cosas?" "Si es que son mis amigos". Y él creía que de esa manera lo que estaba era captando amigos. Él era juguete de otros. ... le pasaban muchas cosas, le tiraban la basura encima, muchas cosas, muchas cosas... (Entrevista a ... madre de usuario). (Negri Cortés, 2016, p. 570).

Apoyándonos en la experiencia narrada por esta madre podemos descubrir que frecuentemente el alumnado con DFI no recibe un trato respetuoso por parte de sus compañeros o compañeras de clase: en muchas ocasiones resultan ser objeto de burlas, insultos e incluso agresiones. Además, puede ser que esta juventud no suela relacionarse con sus iguales en entornos ordinarios, por lo cual resultan especialmente vulnerables y muestran inseguridad ante posibles situaciones de discriminación. Este hecho, unido al gran deseo de estos niños, niñas y jóvenes por la aceptación de su grupo de iguales, puede derivar en la constitución de falsas relaciones de amistad, donde el alumnado con DFl es utilizado para entretenimiento de sus pares.

De estas actitudes podemos deducir que estos compañeros y compañeras no consideran a la juventud con DFI como iguales con los que establecer una relación de amistad basada en el respeto y la igualdad. Para estos grupos, este alumnado se convierte en un objeto pasivo que manejar a su antojo, sin considerar sus sentimientos o las posibles consecuencias de este tipo de actuaciones. Podríamos decir que las relaciones interpersonales que se establecen entre los jóvenes y las jóvenes con DFI y estos compañeros y compañeras siguen un orden jerárquico, donde un grupo goza de una situación de superioridad en cuanto a su situación social respecto del baremo de normalización establecido socialmente. Se crea así una relación desigualdad y de poder, alejada por completo de los principios de inclusión y de igualdad. 
http://doi.org/10.15359/ree.25-3.23

En esta misma línea, cuando preguntamos a profesionales del ámbito socioeducativo si las relaciones personales del alumnado con DFI con sus pares son buenas y se siente integrado en el aula, un 50\% reconoce que sí, pero que suelen tener pocas amistades, seguido de un 30, $6 \%$ que indica que sí, completamente, así como de un 13,9\% que señala que no, no suelen relacionarse con sus compañeros o compañeras, y por último, un 5,6\% admite que no, y además agrega que son objeto de burlas y acoso. Podemos observar la representación gráfica de estos resultados en la Figura 2.

Figura 2: Las relaciones de la juventud con Diversidad Funcional Intelectual y sus compañeros son buenas y se sienten integrados en clase

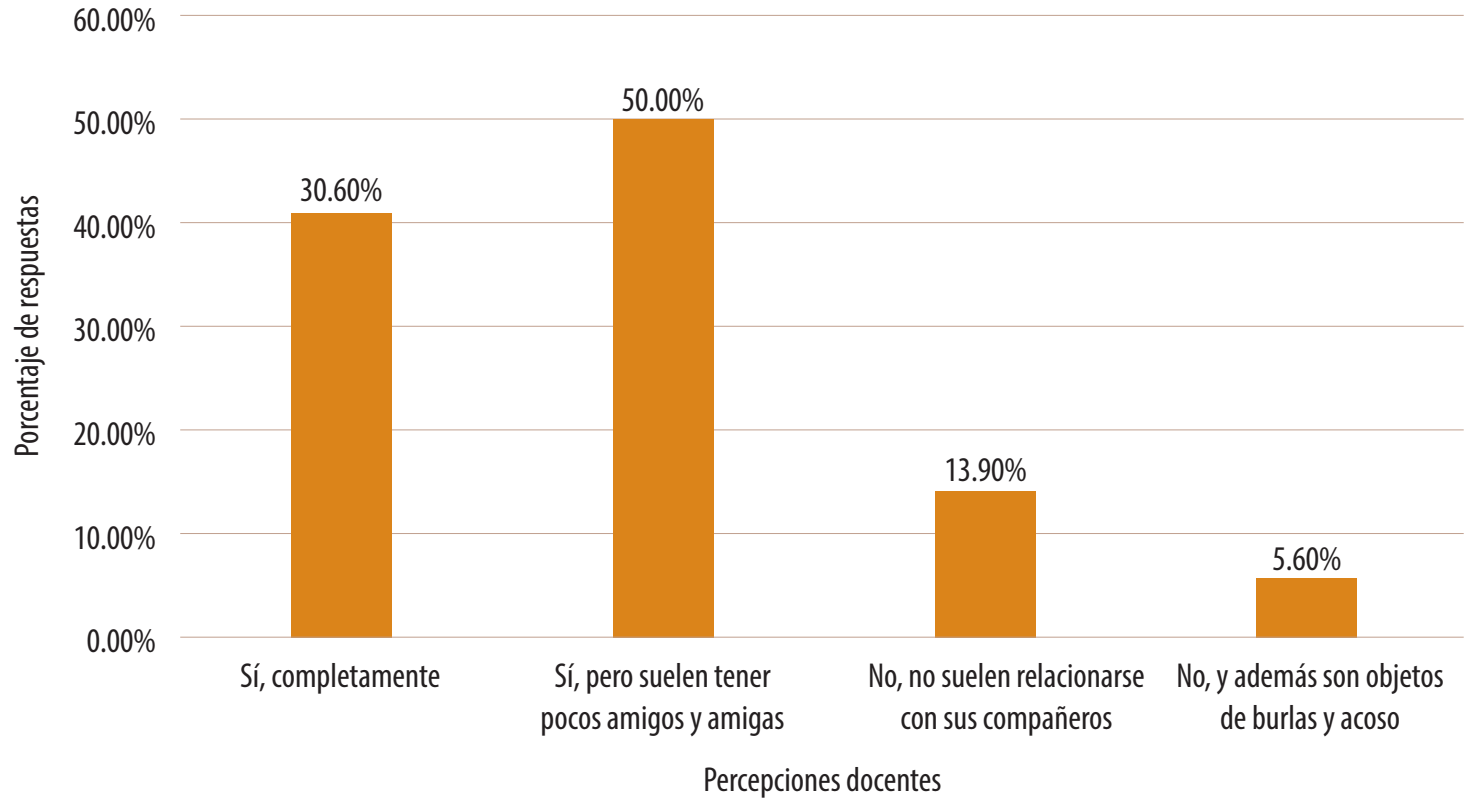

Nota: Elaboración propia.

Estas personas profesionales no parecen percibir situaciones de burlas y acoso hacia la juventud con DFI, pero sí señalan en muchos casos el hecho de que suelen tener pocas amistades. Esta percepción puede tener su origen en una serie de causas: en primer lugar, la asistencia del alumnado con NEE al aula de apoyo a la integración en diversos momentos de la jornada escolar les aísla educativa y socialmente del resto, lo que reduce sus interacciones personales con sus iguales y perpetúa el estereotipo social que define a la DFl; en segundo lugar, en relación con la idea anterior, la juventud con DFI establece sus interacciones sociales y lazos de amistad con otros alumnos y alumnas que pertenecen al mismo grupo de NEE, al asistir en conjunto a los espacios segregadores mencionados. Además, el hecho de compartir su misma condición le otorga una mayor seguridad a la hora de establecer unas relaciones sociales igualitarias; por 
http://doi.org/10.15359/ree.25-3.23

http://www.una.ac.cr/educare

educare@una.ac.cr

último, como consecuencia de los dos aspectos señalados, la percepción que existe sobre la DFI en la sociedad considera a estos grupos de jóvenes como pasivos, infantiles e incapaces, lo cual se refleja en la cultura de los centros educativos, y dificulta su aceptación social.

Por lo tanto, podemos detectar que sí podría existir algún tipo de discriminación en las relaciones personales y sociales que se dan con respecto al alumnado al que nos referimos, aunque no se manifiesten de forma evidente.

\section{Sobre la concepción social de la DFI}

Como hemos mencionado en el apartado anterior, la concepción que presenta la sociedad actual acerca del colectivo al que hace referencia nuestra investigación repercute indiscutiblemente en la ideología presente en los centros educativos y en las actitudes de los miembros y las miembros que participan en los mismos centros. Así pues, si prestamos atención a la visión social que manifiesta el profesorado hacia el alumnado con DFI, debemos observar la siguiente cita:

Entrevistadora: ¿Y el trato de los profesores?

Padre: Yo creo que paternalista, un trato muy...todo el mundo lo quería muchísimo. A mi hijo todo el mundo lo quiere mucho, lo cual no sé si está un poco... Yo creo que el nivel de exigencia era menor que el que debería. Su madre sí le ha tenido un nivel de exigencia en cuestión curricular muy importante. ... Pero, sin embargo, los profesores y las personas que han estado a su cargo pues no han sido exigentes en absoluto, porque es muy buena persona, es muy simpático, muy cariñoso, muy agradable, entonces eso prima sobre cualquier otra cuestión. (Entrevista a padre de usuario de La Unión) (Negri Cortés, 2016, p. 481)

El padre de uno de los usuarios de los programas de inserción socio-laboral de La Unión nos habla de un trato paternalista por parte del profesorado hacia su hijo, basado en la lástima y la sobreprotección, lo cual va en detrimento del desarrollo pleno de las capacidades de este joven. Tanto los aprendizajes académicos como el fomento de la autonomía personal y la independencia social del alumnado deben ir de la mano de exigencias acordes con la edad y el nivel de desarrollo en el que este se encuentre. De lo contrario, existe el riesgo de que la juventud con DFI no sea capaz de desarrollar todas sus potencialidades ni de ejercer sus derechos como personas libres e iguales al resto en una condición de autodeterminación.

$Y$ es que este tipo de actitudes condescendientes perpetúan los comportamientos y actitudes infantiles, haciendo que este alumnado eluda la asunción de responsabilidades propias de su edad y se acomoden a su situación de eternos dependientes.

En relación con la promoción de una atención socio-educativa de carácter inclusivo desde los centros educativos, en nuestro cuestionario se consultó a los grupos profesionales sobre las oportunidades que nos brindan las metodologías de aprendizaje cooperativo en el aula. A tal efecto, de acuerdo con la Figura 3, un $41,4 \%$ señala que estas permiten un aprendizaje más 
http://doi.org/10.15359/ree.25-3.23

enriquecido por las aportaciones heterogéneas del alumnado, mientras un 34,5\% manifiesta que este tipo de estrategia permite una mayor inclusión educativa del alumnado con DFI. Finalmente, un $24,1 \%$ indica que las metodologías de aprendizaje cooperativo mejoran en todo el alumnado la capacidad de trabajo en equipo.

Figura 3: Las metodologías de aprendizaje cooperativo en el aula permiten

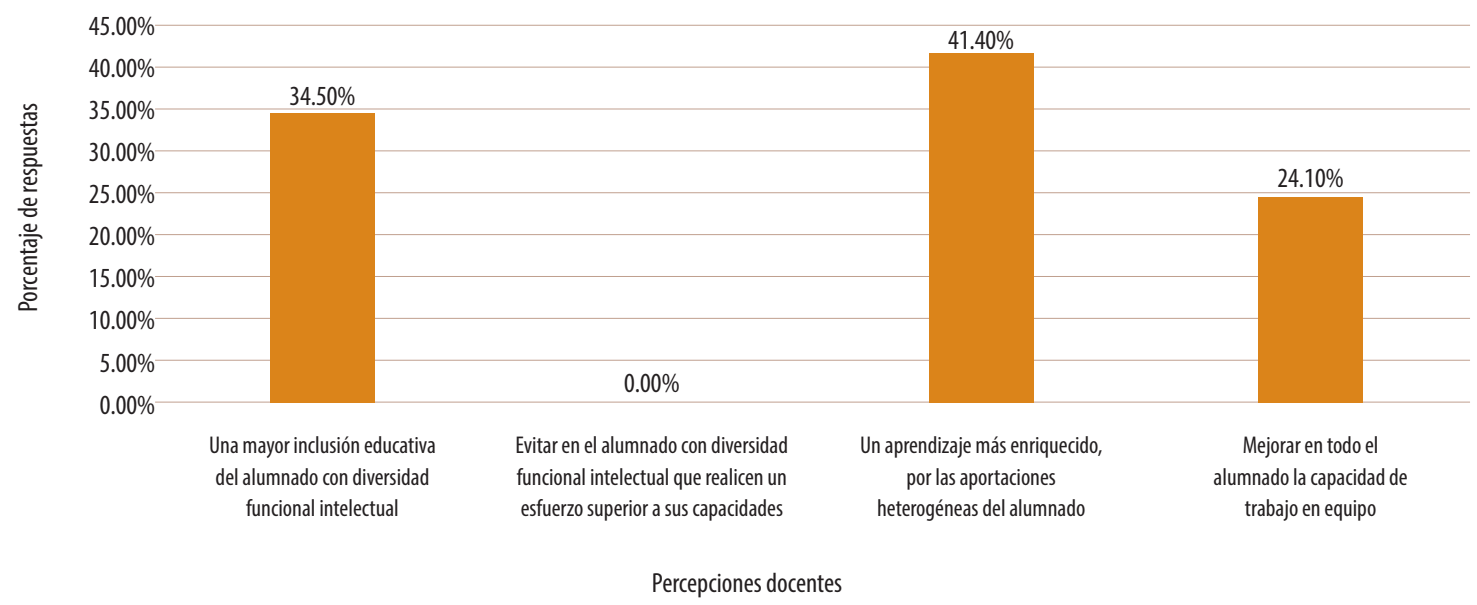

Nota: Elaboración propia.

Los datos obtenidos nos hablan únicamente de las múltiples ventajas que conlleva la utilización de este tipo de metodologías de aprendizaje. Así, la diversidad es entendida como un valor añadido al proceso educativo de todo el alumnado; favorece, además, las interacciones entre todos los miembros y las miembros del grupo clase, lo cual va en beneficio del principio de igualdad de oportunidades. La aplicación de una metodología de aprendizaje cooperativo en el aula contribuye, por lo tanto, a mejorar el proceso de inclusión tanto del alumnado con NEE como de otros alumnos y alumnas que podrían presentar diferentes dificultades personales, familiares y socioculturales. La existencia de un profesorado partidario de la utilización de metodologías de aprendizaje cooperativo se contrapone a las ideas expresadas anteriormente, donde el alumnado con NEE era percibido como una carga para el personal docente. De esta incoherencia podemos deducir la idea de una falta de capacitación del profesorado para atender a las necesidades de todo el alumnado, así como a una falta de sensibilización que rompa con la diferenciación social del alumnado con DFI respecto al resto.

\section{Sobre la autodeterminación}

Las concepciones y actitudes socioeducativas que existen hacia el alumnado con DFI repercuten directamente en la generación de situaciones de rechazo social explícito e implícito. Como consecuencia, son muchos los jóvenes y las jóvenes con DFI que se perciben como personas incapaces y dependientes. 
http://doi.org/10.15359/ree.25-3.23

http://www.una.ac.cr/educare

educare@una.ac.cr

En muchos casos sí, tienen poca autoestima porque si tienen poca discapacidad se dan cuenta de las dificultades que ellos tienen y, por lo tanto, se sienten un poco cohibidos con el resto de la sociedad. Piensan que no van a ser capaces de hacer algunas cosas y tienen normalmente la autoestima un poquito baja normalmente. (Entrevista a ... profesora de taller de PCPI Jardinería). (Negri Cortés, 2016, p. 333)

Podemos deducir que la juventud con DFI asume su papel de rechazada o señalada, llegando a ser conscientes de su diferencia y de la consideración negativa que se le da a esta. De nuevo, esa situación surge del afán de nuestra sociedad por clasificar y diferenciar entre lo que debe ser correcto o normal y lo que no, olvidando que cada ser humano es único y singular. A tal efecto, llegados a cierto nivel de madurez o cognición, la juventud con DFI es consciente de esta distinción y de los prejuicios sociales asociados al colectivo, por lo que una mayoría cuenta con una baja autoestima, que perjudicará su desarrollo autónomo y sus relaciones en los contextos sociales y laborales.

Ahondando en la necesidad de fomentar en la juventud con DFI una buena capacidad para desenvolverse en los diversos contextos ordinarios, hemos consultado a los sujetos participantes de nuestro estudio sobre las habilidades que deben fomentarse en esta juventud a través del uso de metodologías innovadoras. A esta cuestión, según se muestra en la Figura 4, un 44,4\% de las personas profesionales responde que es esencial fomentar en este alumnado la resolución de problemas, seguido de un $33,3 \%$ que apuesta por la capacidad de aprender a aprender, mientras un $11,1 \%$ considera que es preciso potenciar en estos grupos la capacidad de tomar sus propias decisiones y un $7,4 \%$ estima como necesaria la autoestima. Finalmente, un 3,7\% defiende la necesidad de fomentar en este alumnado la capacidad de organizar la información.

Figura 4: El personal docente debe utilizar metodologías que fomenten en la juventud con Diversidad Funcional Intelectual especialmente

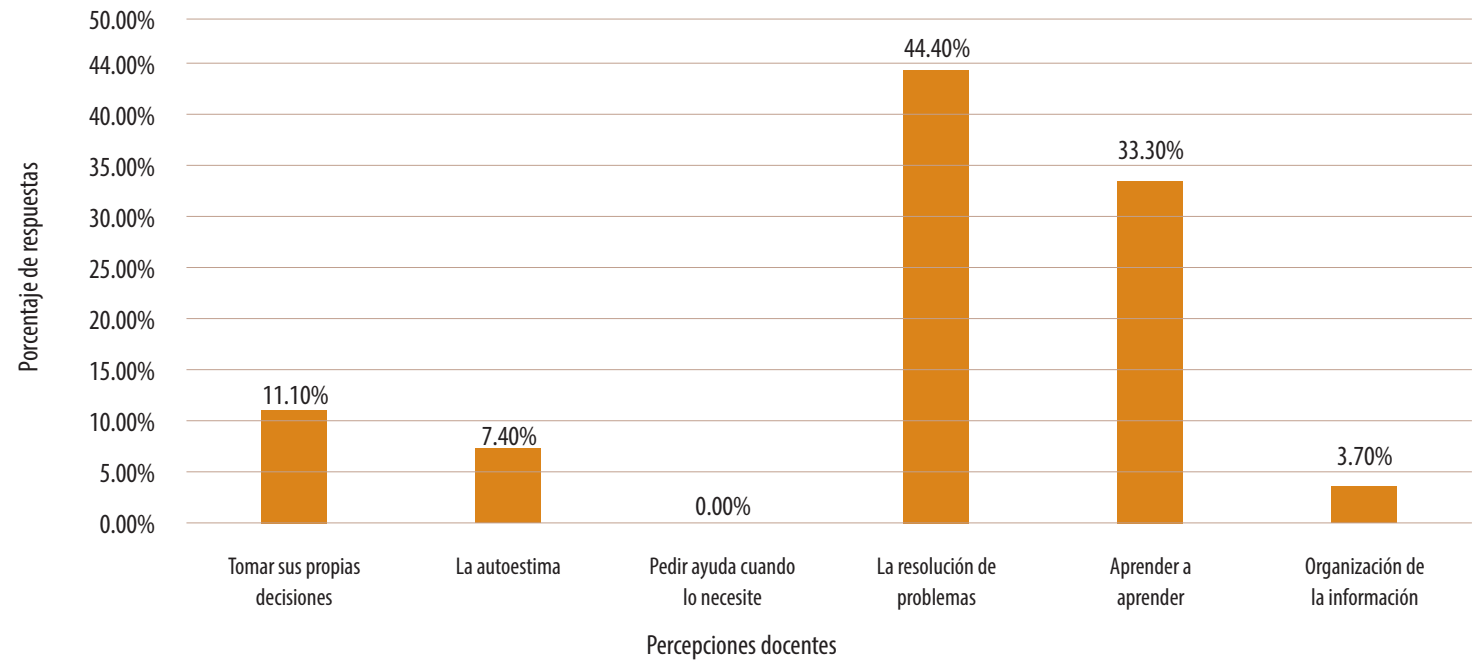

Nota: Elaboración propia. 
Es fundamental, para el grupo de profesionales participantes en esta investigación, que la juventud con DFI tenga una buena capacidad para resolver los problemas de la vida diaria, lo cual, junto a la capacidad de tomar sus propias decisiones y de aprender a aprender, se traduce como un sinónimo de autonomía personal e independencia social. Por tanto, es esencial prestar una educación integral que fomente la capacidad de desenvolverse adecuadamente en los diferentes contextos sociales, y de transferir los conocimientos aprendidos en la escuela a los entornos cotidianos. Por lo tanto, parece adecuado apostar por desarrollar habilidades que fomenten la autodeterminación de la juventud, como las anteriormente citadas.

El desarrollo de la autonomía personal de este alumnado no parece ser una cuestión que preocupe especialmente al personal docente consultado. No obstante, el desarrollo de un alto nivel de autonomía e independencia social favorecerá las relaciones de estos grupos de jóvenes con el entorno, contribuyendo además a su visibilización y a promover una imagen positiva de la DFI. Todo ello, indiscutiblemente, va a influir de manera positiva en su autoestima y autoconcepto, al verse capaces de interactuar de forma igualitaria en los contextos ordinarios.

\section{Discusión}

Hemos visto cómo la juventud con DFI es percibida desde el sistema educativo como un obstáculo que dificulta el buen desarrollo del aula, con base en una serie de parámetros de normalización que aíslan a este alumnado del contexto educativo que sucede dentro de esta misma. La causa de esta problemática, como señalan Negri Cortés y Leiva Olivencia (2019), podría tener su origen en el hecho de que este colectivo durante décadas ha sido invisibilizado en el currículo escolar y en la organización y estructura de los centros educativos. Sin embargo, la solución a esta problemática no puede resultar en delegar todo el peso del desarrollo académico de este alumnado en profesionales de apoyo a la integración.

Al contrario, para solventar este tipo de barreras sería conveniente promover un compromiso por parte de las instituciones educativas, que deberán abogar por la cultura de la diversidad, apostando por la personalización de los aprendizajes y el reconocimiento de la heterogeneidad de todo el alumnado. En estos momentos de debate sobre modificaciones legislativas en materia educativa, puede ser necesario introducir como eje esencial la atención a la diversidad de un alumnado que cumple con las adaptaciones y propuestas curriculares del sistema educativo y que, tras hacerlo, no titula o promociona al contar con dos o más adaptaciones curriculares significativas en materias o áreas instrumentales. Apostar por la práctica real de un currículo común e inclusivo basado en competencias y por una formación permanente del profesorado en metodologías innovadoras y cooperativas de los aprendizajes supone un imprescindible punto de partida para ello. 
http://doi.org/10.15359/ree.25-3.23

http://www.una.ac.cr/educare

educare@una.ac.cr

Hemos conocido las limitaciones que podría presentar el alumnado con DFI en el desarrollo de relaciones de amistad inclusivas, basadas en el respeto y la confianza mutua. Dichas limitaciones pueden surgir de una concepción social negativa de la DFI y del carácter capacitista de los procesos educativos del aula, que sitúan al colectivo por debajo del baremo deseable de normalización, haciendo menos satisfactorio u honorable el hecho de establecer relaciones con estos. Para superar estas discriminaciones y prejuicios hacia la DFI, Luque -Parra y Luque-Rojas (2011) proponen pautas para la intervención en el aula que construyan un adecuado clima de convivencia, que creen una imagen positiva de la DFI y promuevan sus relaciones sociales con el resto de sus compañeros y compañeras. Entre estas pautas, encontramos actuaciones basadas en la aceptación y ayuda espontánea, como la propuesta de tareas de carácter cooperativo, la realización de trabajos específicos en las que colabore alumnado con DFI y sin esta, y relaciones de juego en el aula y patio de recreo, que promuevan la relación de manera natural.

Otra de las conclusiones a las que hemos podido llegar tiene que ver con el trato que otorgan los colectivos profesionales educativos y la sociedad en general a las personas con DFI, el cual viene definido por la sobreprotección e infantilización de estos en detrimento de su desarrollo autónomo. Así, en un estudio desarrollado por Fontana-Hernández y Vargas-Dengo (2018) sobre la inclusión del alumnado con diversidad funcional en el ámbito universitario en Costa Rica, se destaca la idea de que las concepciones sociales sobre este colectivo afectan la percepción que el profesorado tiene sobre las capacidades de estudiantes con esta condición. Al mismo tiempo, Negri Cortés y Leiva Olivencia (2019) señalan la necesidad de promover el liderazgo del personal docente como mediador entre el contexto educativo y social. Podríamos trasladar ambas ideas a la necesidad de promover un cambio ideológico y cultural en el personal docente, que le sensibilice para atender las necesidades reales de este alumnado y responda a su necesidad de aceptación social.

En relación con la dimensión emocional de jóvenes con DFI, observamos que son conscientes de las concepciones y actitudes sociales y educativas que existen hacia ellos y ellas, creándoles inseguridad y bajo autoconcepto. A tal efecto, podemos relacionar esta variable y la autodeterminación del joven y la joven con DFI, y comprender que gracias a esta última podemos mejorar su estado socioemocional. Para la promoción de la autodeterminación del alumnado encontramos herramientas, como el modelo de enseñanza y aprendizaje de la autodeterminación que Mumbardó-Adam et al. (2017) estudian e implementan en España en estudiantes de diferentes características y etapas educativas, entre quienes se incluye la juventud con DFI. Esta práctica, además de trabajar la autodeterminación, mejora el acceso al currículo y la participación en actividades educativas del contexto ordinario, fomenta la enseñanza de habilidades de resolución de problemas y beneficia el establecimiento y consecución de metas personales. La inclusión del alumnado con DFI también debe ir de la mano del fomento de su autonomía e independencia, para lo cual es necesario mantener un nivel de exigencia acorde 
http://doi.org/10.15359/ree.25-3.23

con sus capacidades, que permitirá su crecimiento integral y la adquisición de responsabilidades propias de los contextos sociolaborales.

Como conclusión, la mejora de las concepciones y actitudes socioeducativas hacia la juventud con DFI depende de las sinergias y esfuerzos integrales de todas las instancias y agentes que intervienen en su proceso educativo. Solo así podremos hablar de una sociedad y unas instituciones educativas inclusivas.

\section{Declaración de Material complementario}

Este artículo tiene disponible, como material complementario:

-La versión preprint del artículo en https://doi.org/10.5281/zenodo.4081599

\section{Referencias}

Arnaiz Sánchez, P., de Haro Rodríguez, R. y Azorín Abellán, C. M. (2018). Redes de apoyo y colaboración para la mejora de la educación inclusiva. Profesorado. Revista de Currículum y Formación de Profesorado, 22(2), 29-49. https://doi.org/10.30827/profesorado.v22i2.7713

Barría Rojas, S. y Jurado de los Santos, P. (2016). El perfil profesional y las necesidades de formación del profesor que atiende a los alumnos con discapacidad intelectual en la formación laboral. Profesorado. Revista de Currículum y Formación de Profesorado, 20(1), 287- 310. https://www.redalyc.org/articulo.oa?id=56745576015

Ceballos-Herrera, F. A. (2009). El informe de investigación con estudio de casos. Magis, Revista Internacional de Investigación en Educación, 1(2), 413-423. https://www.redalyc.org/ articulo.oa?id $=281021548015$

Consejería de Educación. (22 de agosto de 2008). Orden de 25 de julio de 2008, por la que se regula la atención a la diversidad del alumnado que cursa la educación básica en los centros docentes públicos de Andalucía. Boletín Oficial de la Junta de Andalucía, 167. https://www.juntadeandalucia.es/boja/2008/167/2

Fontana-Hernández, A. y Vargas-Dengo, M.C. (2018). Percepciones sobre discapacidad: Implicaciones para la atención educativa del estudiantado de la Universidad Nacional de Costa Rica. Revista Electrónica Educare, 22(3), 1-24. https://doi.org/10.15359/ree.22-3.16

García-Ruiz, R. y Sánchez Muñoz, Á. (2012). El aprendizaje de competencias profesionales en los PCPI. Eliminando barreras hacia la inclusión. En F. Guerra López, R. García Ruiz, N. González Fernández, P. Renés Arellano y A. Castro Zubizarreta (Coords.), Estilos de Aprendizaje. Investigaciones y Experiencias, V Congreso Mundial de Estilos de Aprendizaje (pp. 1-6). Universidad de Cantabria. https://dialnet.unirioja.es/servlet/articulo?codigo=4640616 
http://doi.org/10.15359/ree.25-3.23

http://www.una.ac.cr/educare

educare@una.ac.cr

Jefatura del Estado. (4 de mayo de 2006). Ley Orgánica 2/2006, de 3 de mayo, de educación. Boletín Oficial del Estado, núm. 106, pp. 17158-17207. https://www.boe.es/buscar/doc. php?id=BOE-A-2006-7899

Jefatura del Estado. (10 de diciembre de 2013). Ley Orgánica 8/2013, de 9 de diciembre, para la mejora de la calidad educativa. Boletín Oficial del Estado, núm. 295, pp. 97858- 97921. http://www.boe.es/boe/dias/2013/12/10/pdfs/BOE-A-2013-12886.pdf

López, M., Echeita, G. y Martín, E. (2010). Dilemas en los procesos de inclusión: Explorando instrumentos para una comprensión de las concepciones educativas del profesorado. Revista Latinoamericana de Educación Inclusiva, 4(2), 155-176. http://www.rinace.net/rlei/ numeros/vol4-num2/art8.html

Luque-Parra, D. J. y Luque-Rojas, M. J. (2011). Conocimiento de la discapacidad y relaciones sociales en el aula inclusiva. Sugerencias para la acción tutorial. Revista Iberoamericana de Educación, 54(6), 1-12. https://doi.org/10.35362/rie5461640

Macías Gómez, E. (2016). Actitudes de estudiantes de magisterio en educación primaria hacia las personas con discapacidad. Revista Nacional e Internacional de Educación Inclusiva, 9(1), 54-69. https://dialnet.unirioja.es/servlet/articulo?codigo=5455555

Moneo Estany, B. A. y Anaut Bravo, S. (2017). Inclusión del alumnado con discapacidad en los estudios superiores. Ideas y actitudes del colectivo estudiantil. Revista Española de Discapacidad, 5(2), 129-148. https://doi.org/10.5569/2340-5104.05.02.07

Moneo Estany, B. A. y Anaut Bravo, S. (2016). La inclusión socioeducativa en la universidad pública de Navarra. Ideas y actitudes del alumnado hacia sus iguales con discapacidad. En D. Carbonero Muñoz, E. Raya Díez, N. Caparrós Sivera y C. Gimeno Monterde (Coords.). Respuestas transdisciplinares en una sociedad global. Aportaciones desde el trabajo social (pp. 1-11). Universidad de La Rioja. https://publicaciones.unirioja.es/catalogo/online/ CIFETS 2016/Monografia/pdf/TC196.pdf

Mumbardó-Adam, C., Vicente Sánchez, E., Giné I Giné, C., Guardia-Olmos, J., Raley, S. K. y Verdugo Alonso, M. Á. (2017). Promoviendo la autodeterminación en el aula: El modelo de enseñanza y aprendizaje de la autodeterminación. Siglo Cero, 48(2), 41-59. https://doi. org/10.14201/scero20174824159

Negri Cortés, M. I. (2016). Formación e inserción socio-laboral de jóvenes con diversidad funcional intelectual. Una perspectiva pedagógica inclusiva [Tesis Doctoral]. Universidad de Málaga, España. https://riuma.uma.es/xmlui/bitstream/handle/10630/14164/TD NEGRI CORTES Maria Isabel.pdf?sequence=1\&isAllowed=y 
http://doi.org/10.15359/ree.25-3.23

Negri Cortés, M. I. y Leiva Olivencia, J. J. (2019). Liderazgo de los docentes de Formación Profesional Básica para la mediación escuela-empleo del alumnado con diversidad funcional intelectual. Contextos Educativos. Revista de Educación, (24), 95-111. http://doi. org/10.18172/con.3902

Pegalajar Palomino, M. del C. y Colmenero Ruiz, M. de J. (2017). Actitudes y formación docente hacia la inclusión en educación secundaria obligatoria. Revista Electrónica de Investigación Educativa, 19(1), 84-97. https://doi.org/10.24320/redie.2017.19.1.765

Polo Sánchez, M. T. y Aparicio Puerta, M. (2018). Primeros pasos hacia la inclusión: Actitudes hacia la discapacidad de los docentes en educación infantil. Revista de Investigación Educativa, 36(2), 365-379. https://doi.org/10.6018/rie.36.2.279281

Rodríguez González, A. M. (2017). El alumnado de educación secundaria obligatoria con discapacidad intelectual leve en España. Revista Nacional e Internacional de Educación Inclusiva, 10(1), 79-102. https://revistaeducacioninclusiva.es/index.php/REl/article/ view/127/271

Suriá Martínez, R. (2011). Análisis comparativo sobre las actitudes de los estudiantes hacia sus compañeros con discapacidad. Electronic Journal of Research in Educational Psychology, 9(1), 197- 216. https://www.redalyc.org/pdf/2931/293122834010.pdf

Verdugo Alonso, M. Á., Amor González, A. M., Fernández Sánchez, M., Navas Macho, P. y Calvo Álvarez, I. (2018). La regulación de la inclusión educativa del alumnado con discapacidad intelectual:Una reforma pendiente.SigloCero,49(2), 266,27-58. http://dx.doi.org/10.14201/ scero20184922758

Verdugo Alonso, M. Á. y Rodríguez Aguilella, A. (2012). La inclusión educativa en España desde la perspectiva de alumnos con discapacidad intelectual, de familias y de profesionales. Revista de Educación, (358), 450-470. http://doi.org/10.4438/1988-592X-RE-2011-358-086

Vilà Suñé, M., Pallisera Díaz, M. y Fullana Noell, J. (2012). La inclusión laboral de los jóvenes con discapacidad intelectual: Un reto para la intervención psicopedagógica. Revista Española de Orientación y Psicopedagogía, 23(1), 85-93. https://doi.org/10.5944/reop.vol.23. num.1.2012.11396 